(9.)

Nota Técnica | Note

\section{Ecodiseño de una marquesina fotovoltaica para fomentar el uso de las energías renovables en empresas | Eco-}

design of a photovoltaic parking structure to promote the use of renewable energies in companies

\section{Julia Bustillo Erguii, Cristina Alía García² y Alberto Sanchidrián Blázquez²}

scuela Técnica Superior de Ingeneniería en Diseño Industrial. Universidad Politécnica de Madrid. 28012 Madria, Espana Graduada en Ingeniería Mecánica y Diseño Industrial por la Universidad Politécnica de Madrid (UPM). j.bustillo@ alumnos.upmes. ORCID: 0000-0002-1940-6292

a y Diseño Industrial

cristinaalia@upmes ORCID. 0000-0002-2618-0359:

Recibido: 19 de marzo | Aceptado: 10 de mayo | Publicado: 29 de junic

DOl: https://doi.org/10.25267/P56-IDJ.2021.1.7

\section{Resumen}

En 2020 cada vez son más las empresas que buscan fomentar los Objetivos de Desarrollo Sostenibles (ODS) en las políticas que implantan. Destaca la promoción del uso de energías renovables en varios artículos: objetivos número 7, 11 y 13, fundamentalmente. En este contexto se enmarca el mercado de la marquesina fotovoltaica que se plantea en este trabajo. El marketing de la misma se realizará a empresas cuyo interés sea proporcionar a sus empleados un lugar donde poder dejar sus vehículos cargando durante el día laboral, fomentando, además, la sostenibilidad de la propia empresa.

Todo el trabajo se encuadra bajo la metodología del ecodiseño. En este se escogen los materiales y se diseña la estructura para que aporten un valor añadido y reduzcan su impacto medioambiental. Se optimiza el proceso de instalación y montaje para facilitar e proceso de instalación de cara al futuro cliente y disminuir los costes ambientales de esta fase del ciclo de vida de la marquesina. Y por último se diseña un embalaje que permita la comercialización de la marquesina de forma eficaz y que contribuya a la facilidad de su instalacion

Finalmente se evalúan las decisiones tomadas durante el proceso de diseño en función del impacto qué han tenido sobre el producto final, principalmente desde un punto de vista económico, medioambiental y de valor añadido.

Palabras clave: Ingeniería de Diseño, Ecodiseño, Renovable, Diseño Estratégico, Energía solar, Sostenibilida
In 2020, an increasing number of companies are seeking to promote the Sustainable Development Goals (SDGS) in the policies they implement. In this sense, the promotion of the use of renewable energies stands out in several articles: SDGs number 7, 11 and 13 fundamentally. This is the context in which the market for the photovoltaic parking structure proposed in this article is framed. It will be marketed to companies whose interest is to provide their emplovees with a place where they can leave their vehicles charging during the working day, while promoting the sustainability of the company itself.

The whole project is framed under the ecodesion methodology Materials are chosen and The structure is designed to provide added value and reduce its environmental impact. The installation and assembly process is optimized to facilitate the installation process for the future client and to reduce the environmental costs of this phase of the structure's life cycle. Finally, packaging is designed to allow the structure to be marketed efficiently and to contribute to the ease of installation.

Finally, the decisions taken during the design process are evaluated in terms of the impact they have had on the final product, mainly from an economic, environmental and added value point of view.

Key words:

\section{Introducción}

Con el propósito de fomentar los Objetivos de Desarrollo Sostenible (ODS) en las políticas de empresa, las marquesinas fotovoltaicas representan un producto que promocionan estas prácticas: ODS7 (Energía Asequible y No Contaminante), ODS1 (Ciudades y Comunidades Sostenibles) ODS13 (Acción por el Clima) (Programa de las Naciones Unidas para el Desarrollo Objetivos por el Desarrollo Sostenible sf). poresina fotoroltaica consiste una estructura generalmente mecánica que protege a uno o más vehiculos de las inclemencias det tiempo y, además, posee paneles solares en su cu, ademas, posee paneles solares en su cubierta para producir energia. Esta energia puede usarse para cargar los vehiculos o ser usada como consumo habitual.

La mayoria de las marquesinas fotovoltaicas que se encuentran en el mercado siguen medios de montaje profesionales (an Grúas ) y están principalmente diseñadas para satisfacer al mercado empresarial. Se busca optimizar el diseño de la marquesina fotovoltaica tanto para el particular como para la empresa, adoptando la metodología del ecodiseño. El precio de mercado de una marquesina para dos vehículos oscila entre os $7.270 €$ (5.5 kW (Eusion Energía Solar 2021)) a los 13759 , (Fus (37kW. (Solarmat, 2021)), sin incluir montaje ni instalación. embarcarse en una obra de semejante calibre (Peña, 2016)

Una vez definidos los posibles aspectos de meiora se establecen 3 objetivos clave:

1. Elección de la materia prima que permita na mejor integración y una reducción el coste de fabricación y distribución de la marquesina además de su impacto medioambiental, y permita aportar un valor añadido.

2. Optimización del proceso de instalación y montaje de la estructura para evitar costes medioambientales innecesarios, además e reducir las molestias ocasionadas al cliente. Además, se suma el esfuerzo que supone 
3. Diseño de un embalaje que permita la comercialización de la marquesina de forma eficaz, disminuyendo or impacto de su transporte y facilitando el montaje.

El diseño de la marquesina fotovoltaica se encuadra bajo la metodología del ecodiseño "El ecodiseño también conocido como diseño para el medio ambiente, es una metodología que considera la variable ambiental como un criterio mas en el proceso de diseño de productos industriales, sumándose asía otros factores previos com los costes económicos o la calidad" (ihob 2021). Esta metodología busca reducir el impacto medioambiental de la marquesina en todas las fases de su vida útil, desde el diseño hasta la comercialización, pasando por la producción, transporte y montaje.

\section{Metodología}

Se parte de una estructura de perfiles normalizados de acero, que tiene cabida para 2 vehiculos y que sostiene 15 módulos solares de 2 m2 cada uno. Este tipo de estructuras pueden encontrarse ya en el mercado pero tienen un amplio rango de mejora El primer cambio propuesto es sustituir el acero por aluminio.

Los perfiles de aluminio tienen múltiples ventajas sobre los perfiles tradicionales de acero, como su menor densidad (lo que facilita su transporte y montaje), su facilidad de reciclaje o su larga vida útil (Diferencias entre el aluminio y el acero, 2021).

Como parte del proyecto se rediseña la tipología de la marquesina. Se opta por reducir el ángulo de la cubierta de $20^{\circ}$ rvar más cercano a la inclinación idónea cerce de (Madid) a $10^{\circ}$. Por otro lado optimiza la estructura, evitando e uso de perfites auxilares de fuacion para la instalación solar, y en su lugar se situan las correas a una distancia entre especifica que permite la fijación de los módulos solares directamente a los perfiles preexistentes.
Por ultimo, para facilitar el transporte se dividen los perfiles en dos piezas, de modo quela lonsitud maxima del mayorde olloses de 2,92 m (para et caso de la viga principal). Estas partes se vuelven a unir en el proceso de montaje. En la Figura 1 se pueden ver lónos ejemplos de las uniones diseñadas para el nexo en el centro de estas vigas.

Figura 1. Unión central en el pilar de mayor longitud (a) yunión central de la viga principal (b)

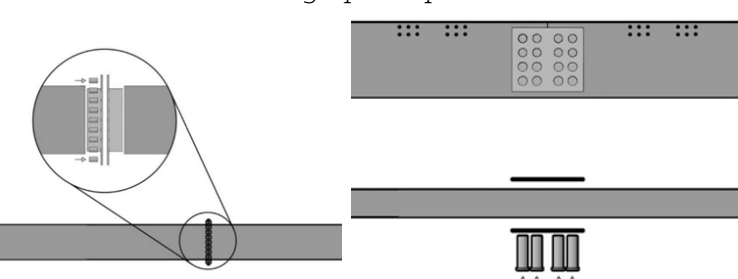

Resultados

El estudio estructural con perfiles de aluminio demuestra que los perfiles necesarios dados los esfuerzos que recibe la estructura son relativamente pequeños. Gracias a esto la estructura puede resolverse usando perfiles huecos rectangulares de tan bien a flexión como los trabefiles normatizados de acero (IPE, HEB.). Estos cambias pueden a Tabla 1 a continuación, que compara los perfiles usados en una estructura de perfiles normalizados de acero de $20^{\circ}$ de angulo de cubierta con una estructura de perfiles rectangulares huecos de aluminio de $10^{\circ}$ de angulo de cubierta

Tabla 1. Comparativa estructural de los perfiles de acero y aluminio

Se observa que el precio de la estructura de aluminio desciende un 14\% y el peso un $47 \%$ respecto a la estructura de acero. La disminución del peso es correlativa con la disminución de los gases de efecto invernadero generados durante el transporte, obteniendose un beneficio medioambiental.
El cambio en el ángulo de la cubierta supone una disminucion de la produccion solar del 32\%, pero favorablemente reduce los efectos del viento del $26 \%$, permitiendo el uso de perfiles de menor dimension reduciendo el volumen de la cimentación en un $30 \%$.

l uso de perfiles rectangulares huecos facilita las uniones entre las diferentes vigas y pilares. Como parte del objetivo de optimizacion de la instalación y montaje de la estructura, ésta se diseña de forma que se pueda plegar sobre plano y elevar manulalmente mediante la ayuda de un "tractel". Para poder ir desplegando la "estructura es necesario que los perfiles que forman los plares y la viga que recibe puedan grar relativamente puro se faclita usando perites ta usando perfiles rectangulares huecos, ya que solo es necesario añadir una pieza auxiliar de giro mecanizar uno de los perfiles para permiti el giro relativo entre sí. Un ejemplo de este tipo de union se puede ver en la Figura 2

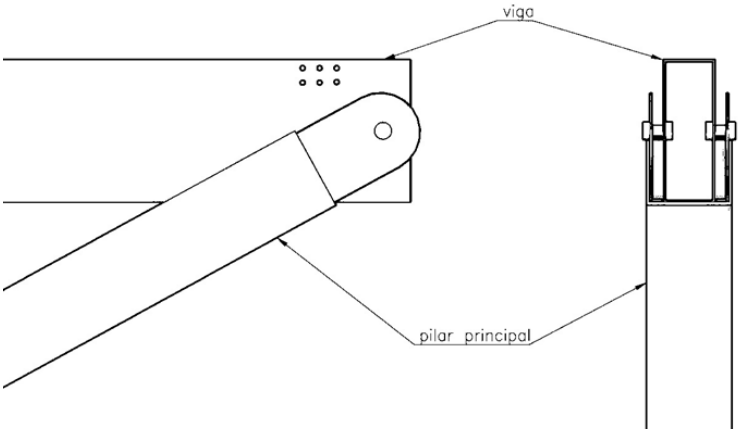

Figura 2. Unión entre la viga principal y uno de los pilares

La sencillez de la unión optimiza tanto el tempo de montaje como el de los traslados de montadores. Generando un importante beneficio tanto económico como ambiental. Este cambio permite el montaje al propio cliente sin necesidad de grandes medios auxiliares, ni conocimientos tecnicos.

El diseño de un embalaje que facilite el transporte y la comercialización de la marquesina es un aspecto clave para reducir emisiones. El dividir los perfiles en dos piezas se consigue un embalaje manejable
$(88 \mathrm{~cm} \times 122 \mathrm{~cm} \times 304 \mathrm{~cm})$ y cada perfit puede ser manipulado entre dos personas. El embalaje esta diseñado para transportar dos estructuras simultáneamente en un uroón de caróa ligera reduciendo el impacto negativo del transporte a la mitad. Los tres montadores necesarios para montar la estructura se desplazan en el vehículo.

En la Figura 3 puede verse como se embalan as dos estructuras en cajas de madera sobre un palet europeo, que se depositan en a zona de carga del furgón. Adicionalmente se cargan los paneles solares necesarios para cada instalación Optimizando de esta manera el transporte de la marquesina al completo.

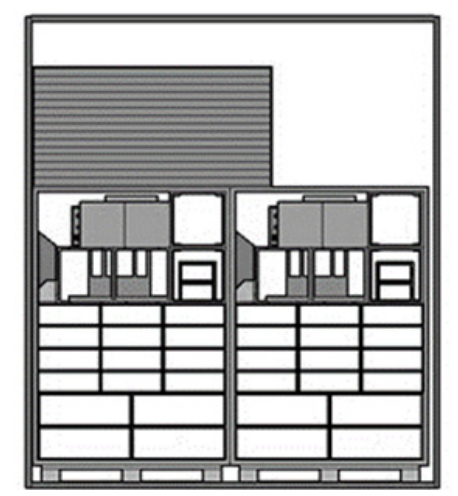

Figura 3. Embalaje de dos estructuras en caja de madera sobre palet europeo

a estructura final es sencilla, de líneas mpias y con unfuerte componente estético o que ayuda a diferenciarse en un mercado en el que ya existen las marquesinas otovoltaicas. Por otro lado, el concepto modular facilita la integración de esta estructura ya que permite comercializar ta misma estructura para el cliente individual misma estructura para el ciente indidualy l número de módulos que desea así como permitir futuras extensiones.

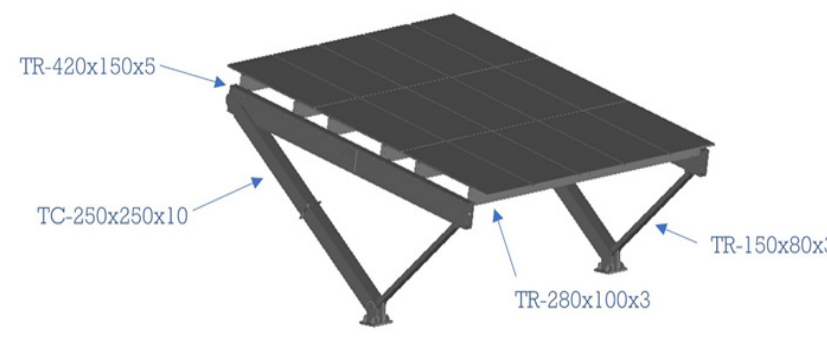

Figura 4. Estructura final de la marquesina fotovoltaica 


\section{Conclusiones}

Los resultados obtenidos durante el proceso son positivos en todos los aspectos Se logra disminuir el impacto medioambiental de la estructura y se consigue una reducción de os costes generales de la marquesina. La larga vida útil de la estructura de aluminio, gracias a su resistencia a la corrosión, frente a la de los paneles solares, 25 años, conlleva a posibilidad de sustituir los paneles solares una vez se acabe su vida útil. Una vez se leóne al fin de esa vida útil, la estructura de aluminio es fácil de desmontar, at ser todas sus uniones atornilladas. De ese modo se facilta el recictaje de tas diferentes partes de la estructura ya que al reciclar aluminio se ahorra el 95\% de la enerǵa que sería necesario para extraerlo desde mineral. Otro beneficio añadido de realiza la estructura en aluminio es que este material es resistente a la corrosión, lo que facilita el mantenimiento de la estructura a no requerir una imprimacion de pintura suponiendo un beneficio tanto a nive práctico como medioambiental.

instalar una marquesina solar en una empresa fomentaría y ayudaría a los empleados a tener un vehículo eléctrico sin aumentar su consumo energetico. Es más, el excedente producido podría ser aprovechado por la red de la empresa. A un particular le permite proteger su vehículo y. paralelamente, gonerar electricidad para su hogar

En conclusión, los impactos sociales y medioambientales de este proyecto son positivos, ya que gracias a esta marquesina se fomenta el uso de vehículos eléctricos se aumenta la cantidad de energía enovable que se produce. La instalación de la marquesina supone un ahorro economico para el consumidor a medio y largo plazo.

\section{proyecta 56}

Diferencias entre el aluminio y el acero. Febrero de 2021). (Perfiles de Aluminio. Net) Recuperado el 10 de 2020, de https: perfilesdealuminio net/articulo/diferencias entre-el-aluminio-y-el-acero/21

Fusion Energía Solar. (Marzo de 2021) Recuperado el 21 de 09 de 2020, de https:/fusionenergiasolar.es/kits-solaresbaratos/466-kit-solar-con-marquesinapara-recarga-de-2-coches-electricos-4tw html

ihobe. (03 de 2021). Obtenido de https: wWW.ihobe.eus/ecodisen

Peña, G. M. (26 de Septiembre de 2016). Houz2 Obtenido de https.//www hourzes/revista/esposible-convivir-con-una-obra-en-casastsetivys-vs 72638080

Programa de las Naciones Unidas para l Desarrollo - Obietivos por el Desarrollo Sostenible. (s.f).). (Naciones Unidas) Recuperad. el 10 de 2020, de https://www:undp.org/content undp/es/home/sustainable-developmentgoals.htm

Solarmat. (Marzo de 2021) Obtenido de https://solarmates/es/marquesinas- solares/ marquesina-solar-monoposte-pvs-2

plazas-trifasica.html

\section{Contribución de autores.}

Conceptualización, J.B.-E., C.A.G. y A.S.B. netodologia, J.B.-E; software, J.B.-E. y A.S.B; alidacion, J.B.-E. y A.S.B; analisis formá, documento orioinal JB.-E: revisión édición J.B.-E..C.A.G. y A.S.B; supervisión, Y A.S.B. Todos los autores han leído y stán de acuerdo en publicar esta versión uente de financiación.
Revisión | Review

Revisión de los nuevos planteaminetos de diseño para conseguir estrategias de Zero Waste

\section{Marta Gómez-Martinez}

Calot 3-7, Igualada

design.martagomez@gmal.com

Recibido: 19 de marzo |Aceptado: 18 de mayo | Publicado: 29 de junio

Dol: https://doi.org/10.25267/P56-IDJ.2021.1.2

\section{Resumen}

Desde el último tercio del siglo XX se ha generado una conciencia social en torno al desarrollo sostenible que se ha visto reflejado por medio de los diferentes pactos, acuerdos y normas que fomentan la economía circular. con los nuevos planteamientos en las estrategias de diseño hacia el Zero Waste, se pueden alcanzar soluciones respetuosas con el medio ambiente que no sólo cumplan los diferentes objetivos nacionales e internacionales propuestos por la ONU, sino que permitan una evolución social haciendo uso de los recursos actuales reduciendo la huella de carbono, el impacto ambiental y la utilización de recursos limitados, entre otros

Analizando a diversos autores, se definen los principales términos para llevar a cabo el objetivo de esta revisión, junto con el estudio de la comparativa de los diferentes modelos y filosofías antecesores que ejercen de premisa a la economía circular, el Zero Waste y el desarrollo sostenible. Estudiando los indicadores mencionados de las diferentes fases que conforman el proceso conceptual y de fabricación, y junto con la distribución del propio producto hasta el final de su vida, se examinan, las diferentes estrateglas usadas actualmente en el sector

El uso de las nuevas tecnologías, la investigación de los nuevos materiales experimentales y las innovaciones en el campo del diseño prometen un nuevo concepto de producto y estilo de vida eco-sostenible que permite reducir e incluso eliminar tanto el impacto ambiental como la huella de carbono.

Reciclando, reutilizando y reacondicionando se puede lograr acabar con los despilfarros y desechos, reduciendo así la fabricación de nuevos productos, alcanzando una producción optima que no ponga en peligro el futuro de la humanidad apostando por un producto local, de cercaniay sostenible.

Palabras clave: Diseño Industrial, Diseño de Producto, Ingeniería de diseño, Ciencia del Diseño, Sostenibilidad, cero desperdicios.

\section{Abstract}

